\title{
Observation of Magnetic Circular Dichroism in the Electron Microscope
}

C cileH ber*, S. Rubino*, P. Schattschneider*, J. Rusz**, P. Novak ${ }^{* *}$, C. Hurm ${ }^{* * *}$, J. Zweck***

* Institut $\mathrm{f} r$ Festk rperph ysik, TU Wien, Wiedner Hauptstra e 8-10 1040 Vienna, Austria

** Institute of Physics ASCR, Cukrovarnick 10, 16253 Praha 6, Czech Republic

*** Institute for Magnetism and Magnetoelectronics, University of Regensburg, D - 93040 Regensburg, Germany

An important tool in the study and characterization of magnetic materials is the synchrotron based technique called X-ray Magnetic Circular Dichroism (XMCD), in which the intensities of the absorption spectral lines vary with the helicity of the circularly polarized exciting radiation.

For many years already, Linear Dichroism experiments have been performed in the Transmission Electron Microscope (TEM) where they are known as orientation-dependent variation of spectral line shape in anisotropic materials. However, to measure circular dichroism in the TEM was thought to be related to the possibility to obtain a spin-polarized beam of electrons. Only in 2003 it was demonstrated theoretically that this is not the case, as the equivalent of a circularly polarized photon can be obtained from the interference of two coherently scattered electron beams dephased by $\pi / 2$ [1]. An experimental veri cation of the effect (named Energy loss Magnetic Chiral Dichroism, EMCD) was recently obtained with TEM and synchrotron measurements on the same Fe specimen [2]. Several experimental setups based on the principle of angle resolved Electron Energy Loss Spectrometry (EELS) allow to record a chiral dichroic signal in the TEM. Measurements can be done in image mode or in diffraction mode, using an imaging lter or a spectrometer. The choice of the experimental setup in uences the achievable spatial resolution as well as the signal to noise ratio. Either a biprism or the (crystal) target itself can be used as beam splitter. In the experiment, a coherent superposition of two momentum transfer vectors perpendicular to each other is set up, tuning the phase difference between the two interactions to $\pi / 2$. The inelastic interference term carries the dichroic signature. Experimental details and recent results on $\mathrm{Ni}$ and Co will be presented, as well as simulations. Calculations were done with a full-potential, fully-relativistic Augmented Plane Wave code based on Density Functional Theory. A good approach to the understanding of EMCD is the mixed dynamic form factor. Chiral dichroism shows up as an imaginary part of the MDFF whereas linear dichroism is equivalent to the anisotropy of the dynamic form factor. Of particular interest are $\mathrm{L}_{2,3}$ or $\mathrm{M}_{4,5}$ ionisation edges of atoms with magnetic moments. The signal in X-ray absorption spectra depends on the orientation of the atomic magnetic moment relative to the photon's wave vector, and on its chirality. Similarly, the ne structure (ELNES) in an EMCD experiment depends on the orientation of the atomic magnetic moment relative to the incident electron's wave vector (for small energy losses), and on the phase shift mentioned above. The EMCD technique provides a new analytical tool for the element speci $\mathrm{c}$ study of local magnetic moments on a nanometre scale. Applications cover magnetic ordering, spin and orbital magnetization, and electronic correlation, e.g. in heavy fermion systems. The TEM may thus complement the synchrotron for the study of magnetic properties in technologically relevant materials.

References

[1] C. H bert,P. Schattschneider, Ultramicroscopy 96 (2003) 463.

[2] P. Schattschneider et. al., submitted to Nature .

[3] This research was supported by the European Commission, contract nr. 508971(CHIRALTEM). 


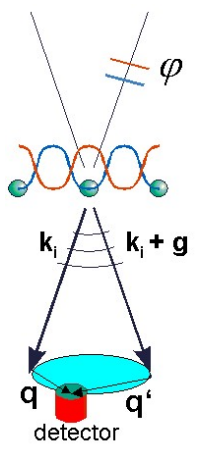

Contrast Aperture or detector

(000)

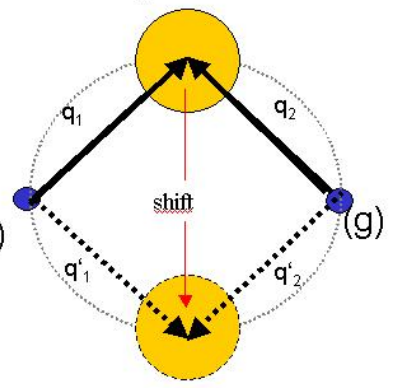

Fig. 1: Scattering geometry. Either the detector or an aperture are used to select the nal wave vector $\overrightarrow{k_{f}}$ (and therefore the momentum transfers $\vec{q}$ and $\left.\overrightarrow{q^{\prime}}\right)$. The dotted circle represents the points for which $\vec{q} \perp \overrightarrow{q^{\prime}}$. The full circles show the two positions for which also the condition $q \approx q^{\prime}$ is true and indicate the actual experimental setup. As the two positions have oppose chirality, EMCD can be detected by simply acquiring spectra at the two positions and taking their difference.
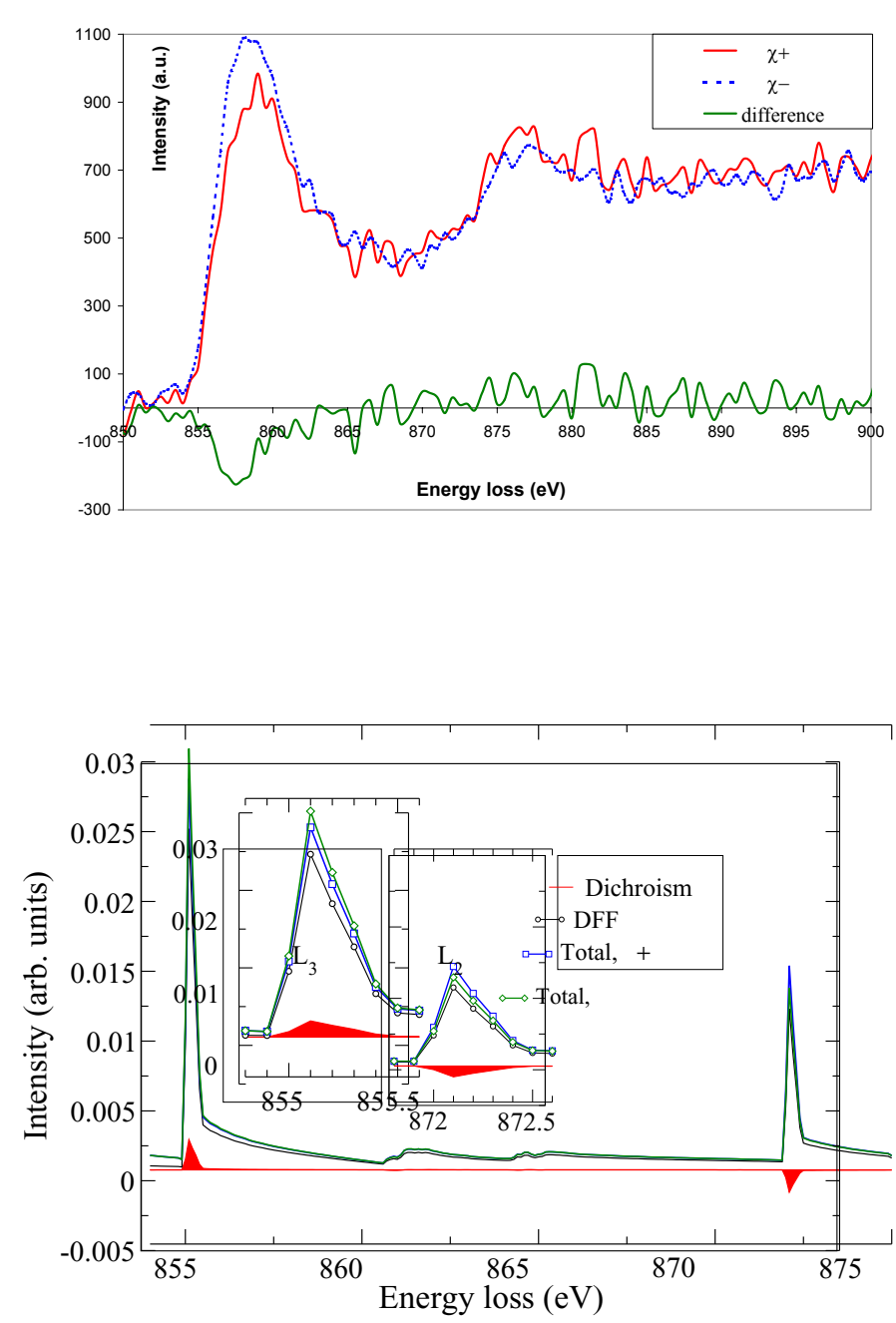

Fig. 2: Experiment. $\mathrm{L}_{23}$ spectra measured on a $100 \mathrm{~nm}$ radius monocrystalline region of a $50 \mathrm{~nm}$ thick Ni specimen for the two detector positions shown in Fig. 1, and the difference (EMCD) signal.

Fig. 3: Simulation. Calculated spectra and EMCD signal ( lled areas) for the scattering geometry of Fig. 1. No braodening has been applied to the spectra. Therefore the ratio between the white lines ( $\mathrm{p} \rightarrow \mathrm{d}$ transitions) and the continuous background appears higher than in the experiment. Inserts show details of the white line regions. 